II presente documento viene fornito attraverso il servizio NILDE dalla Biblioteca fornitrice, nel rispetto della vigente normativa sul Diritto d'Autore (Legge n.633 del 22/4/1941 e successive modifiche e integrazioni) e delle clausole contrattuali in essere con il titolare dei diritti di proprietà intellettuale.

La Biblioteca fornitrice garantisce di aver effettuato copia del presente documento assolvendo direttamente ogni e qualsiasi onere correlato alla realizzazione di detta copia.

La Biblioteca richiedente garantisce che il documento richiesto è destinato ad un suo utente, che ne farà uso esclusivamente personale per scopi di studio o di ricerca, ed è tenuta ad informare adeguatamente i propri utenti circa i limiti di utilizzazione dei documenti forniti mediante il servizio NILDE.

La Biblioteca richiedente è tenuta al rispetto della vigente normativa sul Diritto d'Autore e in particolare, ma non solo, a consegnare al richiedente un'unica copia cartacea del presente documento, distruggendo ogni eventuale copia digitale ricevuta.

Biblioteca richiedente: Biblioteca Dipartimento Scienze di base e applicate per l'ingegneria - Sez.Fisica

Data richiesta:

04/11/2020 10:48:20

Biblioteca fornitrice: INAF - Osservatorio Astronomico di Roma

Data evasione: $\quad$ 04/11/2020 19:34:50

Titolo rivista/libro: $\quad$ Proceedings of SPIE

Titolo articolo/sezione: Design and testing of ultrafast plasmonic lens nanoemitters

Autore/i: $\quad$ Durham,Rotta Loria, Riminucci

ISSN: $\quad 1996-756 X$

DOI:

Anno:

Volume:

11462

Fascicolo:

Editore:

Pag. iniziale:

Pag. finale: 


\section{Design and testing of ultrafast plasmonic lens nanoemitters}

Durham, Daniel, Rotta Loria, Silvia, Riminucci, Fabrizio, Kanellopulos, Kostas, Shen, Xinglai, et al.

Daniel B. Durham, Silvia Rotta Loria, Fabrizio Riminucci, Kostas

Kanellopulos, Xinglai Shen, Filippo Ciabattini, Andrea Mostacci, Pietro

Musumeci, Andrew M. Minor, Stefano Cabrini, Daniele Filippetto, "Design and testing of ultrafast plasmonic lens nanoemitters," Proc. SPIE 11462,

Plasmonics: Design, Materials, Fabrication, Characterization, and Applications XVIII, 1146222 (20 August 2020); doi: 10.1117/12.2567540

SPIE. Event: SPIE Nanoscience + Engineering, 2020, Online Only 


\title{
Design and testing of ultrafast plasmonic lens nanoemitters
}

\author{
Daniel B. Durham ${ }^{\mathrm{a}, \mathrm{b}}$, Silvia Rotta Loria ${ }^{\mathrm{c}, \mathrm{d}}$, Fabrizio Riminucci ${ }^{\mathrm{d}, \mathrm{e}}$, Kostas Kanellopulos ${ }^{\mathrm{d}, \mathrm{f}}$, \\ Xinglai Shen ${ }^{\mathrm{g}}$, Filippo Ciabattini ${ }^{\mathrm{h}}$, Andrea Mostacci ${ }^{\mathrm{i}}$, Pietro Musumeci ${ }^{\mathrm{g}}$, Andrew M. Minor ${ }^{\mathrm{a}, \mathrm{b}}$, \\ Stefano Cabrini ${ }^{\mathrm{e}}$, and Daniele Filippetto ${ }^{\mathrm{j}}$ \\ ${ }^{a}$ Department of Materials Science and Engineering, University of California, Berkeley, \\ Berkeley, California 94720, USA \\ ${ }^{b}$ National Center for Electron Microscopy, Molecular Foundry, Lawrence Berkeley National \\ Laboratory, One Cyclotron Road, Berkeley, California, 94720, USA \\ cPolytechnic University of Milan, Piazza Leonardo da Vinci, 32, 20133 Milan, Italy \\ dMolecular Foundry, Lawrence Berkeley National Laboratory, One Cyclotron Road, Berkeley, \\ California, 94720, USA \\ ${ }^{\mathrm{e}}$ Department of Mathematics and Physics, University of Salento, Campus Ecotekne, 73100 \\ Lecce, Italy \\ ${ }^{f}$ Polytechnic University of Turin, Corso Duca degli Abruzzi, 24, 10129 Turin, Italy \\ ${ }^{g}$ Department of Physics and Astronomy, University of California, Los Angeles, Los Angeles, \\ California, 90095, USA \\ ${ }^{\mathrm{h}}$ Department of Information Technology and Electrical Engineering, ETH Zurich, CH-8092 \\ Zurich, Switzerland

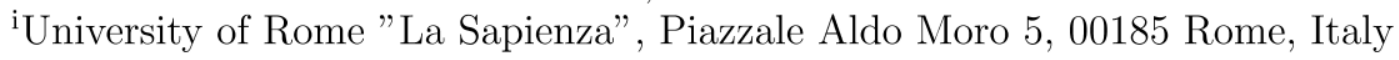 \\ ${ }^{j}$ Accelerator Technology and Applied Physics Division, Lawrence Berkeley National \\ Laboratory, One Cyclotron Road, Berkeley, California, 94720, USA
}

\begin{abstract}
Nanoscale electron pulses are increasingly in demand, including as probes of nanoscale ultrafast dynamics and for emerging light source and lithography applications. Using electromagnetic simulations, we show that gold plasmonic lenses as multiphoton photoemitters provide unique advantages, including emission from an atomically flat surface, nanoscale pulse diameter regardless of laser spot size, and femtosecond-scale response time. We then present fabrication of prototypes with sub-nm roughness via e-beam lithography, as well as electro-optical characterization using cathodoluminescence spectromicroscopy. Finally, we introduce a DC photogun at LBNL built for testing ultrafast photoemitters. We discuss measurement considerations for ultrafast nanoemitters and predict that we can extract tens of pA photocurrent from a single plasmonic lens using a Ti:Sa oscillator. Altogether, this lays the groundwork to develop and test a broad class of plasmon-enhanced ultrafast nanoemitters.
\end{abstract}

Keywords: Plasmon-enhanced photoemission, plasmonic lenses, ultrafast optics, nanoplasmonics, multiphoton photoemission, cathodoluminescence spectromicroscopy, ultrafast electron microscopy, electron-beam lithography

\section{INTRODUCTION}

Electron beams are valuable tools for science and industry. Electron microscopes image and probe the structure, chemistry, and properties of matter with nanoscale or even atomic resolution. Large-scale scientific facilities like accelerators, synchrotrons, and free-electron lasers utilize high-energy electron beams to study high-energy

Further author information: (Send correspondence to D.F.)

D.F.: E-mail: dfilippetto@lbl.gov

D.B.D.: E-mail: dbdurham@berkeley.edu

Plasmonics: Design, Materials, Fabrication, Characterization, and Applications XVIII, edited by Din Ping Tsai, Takuo Tanaka, Proc. of SPIE Vol. 11462, 1146222

(C) 2020 SPIE · CCC code: $0277-786 X / 20 / \$ 21 \cdot$ doi: $10.1117 / 12.2567540$ 
physics or generate bright photon probes. Electron-beam lithography tools enable patterning of nanometric features not yet achieved with photolithography.

Emerging applications for electron beams demand temporal control in addition to spatial control. For instance, ultrafast electron microscopes ${ }^{1}$ and diffractometers ${ }^{2}$ are being developed to view nanoscale dynamics with picosecond or faster time resolution. Free-electron lasers use bright electron pulses to induce the self amplification of stimulated emission. ${ }^{3}$ Techniques for multibeam electron-beam lithography are being developed to parallelize the technique for increased throughput. ${ }^{4}$ Photoemitters are being used or developed for all of these applications to take advantage of the temporal precision and shaping afforded by lasers and optics.

In recent years, plasmonics has been increasingly explored as a means to enhance photoemission. For instance, gratings have been patterned into the sides of tip emitters to convert incident laser pulses to surface plasmon polaritons focused at the apex, providing efficient conversion to photoelectrons. ${ }^{5}$ Metallic photocathodes have been patterned with line ${ }^{6,7}$ and round hole gratings ${ }^{8}$ to enhance absorption and photocurrent by orders of magnitude. Still, much of the potential of plasmon-enhanced photoemission has not been exploited; intelligent design of plasmonic nanostructures allows detailed manipulation of the spatial and temporal profiles of optical excitation and, consequently, photoemitted electron beams.

We are studying the application of plasmonic lenses as pulsed electron nanoemitters. Our electromagnetic simulations show that through a combination of surface plasmon polariton focusing and multiphoton photoemission, gold plasmonic lenses can achieve sub-100 nm emitted spot size with sub-10 fs temporal response. We have fabricated large arrays of high-quality plasmonic lenses using electron-beam lithography and verified their plasmonic properties using cathodoluminescence spectromicroscopy. We also recently built a DC photogun to test photoemission of these and other next-generation photoemitters with demonstrated specifications for measuring the emitted electron beams. This work is paving the way for a series of future developments in ultrafast nanoemitter design and testing.
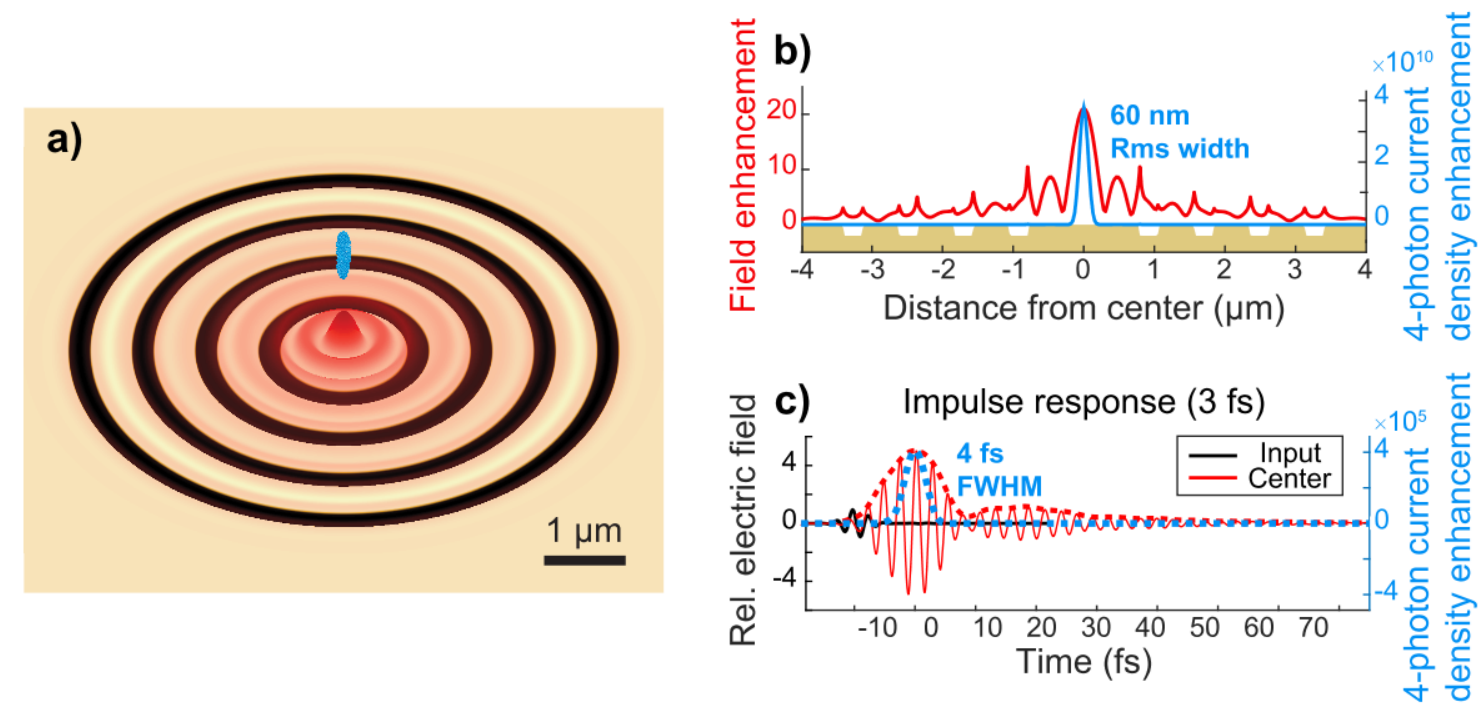

Figure 1. Design and simulation of plasmonic lens emitter. (a) Illustration of concept and geometry. Simulated electric field distribution is shown in red as well as a cartoon emitted electron bunch in blue. Grooves (black) are $90 \mathrm{~nm}$ deep and $270 \mathrm{~nm}$ wide with $783 \mathrm{~nm}$ period. (b) Simulated electric field profile using a radially polarized $800 \mathrm{~nm}$ wavelength laser using 0.07 numerical aperture. The four-photon current density is superimposed, computed as eighth power of the field. (c) Simulated impulse response given relative to the maximum input field using a 3-fs FWHM laser pulse. 


\section{PLASMONIC LENS EMITTER PRINCIPLES}

Plasmonic lens emitters achieve nanoscale concentration of optical fields with femtosecond response times using surface plasmon polariton (SPP) interference. Annular gratings with periodicity of the SPP wave vector convert normally incident light into SPPs,${ }^{9}$ half of which travel towards the center of the structure and interfere. ${ }^{10}$ One plasmonic lens design we are studying is a gold bullseye plasmonic lens as shown in Figure 1a. ${ }^{11}$ In this case, radially polarized laser beams provide optimal excitation: they have polarization vectors perpendicular to the grating at all azimuthal angles, thereby maximizing the energy coupled into SPPs and constructive interference at the center. ${ }^{12,13}$ For the bullseye lens design shown, this interference leads to the spatial optical field profile shown in Figure 1b and temporal impulse response shown in Figure 1c, as determined by electromagnetic simulations performed in Lumerical. ${ }^{14}$ The short temporal response is the consequence of utilizing interfering SPPs rather than surface plasmon resonance, although weak cavity resonance is present leading to some ringing in the temporal response. The full details of these electromagnetic simulations are presented in ref. 11.

This gold plasmonic lens emitter design exploits multiphoton photoemission to achieve small, short electron pulses. The example shown is for a laser with $800 \mathrm{~nm}$ wavelength, in which case four photons are required to extract one electron. The scaling of n-photon photocurrent with the nth power of the electric field intensity suppresses weaker side features in the profiles and shrinks the peak widths, leading to $60 \mathrm{~nm}$ root-mean-square (RMS) emitted spot size and 4 fs full width at half maximum (FWHM) photoemission duration. Concentration of optical intensity into femtosecond, nanoscale pulses improves the probability of such a 4-photon process by orders of magnitude, as will be further explored in section 4.
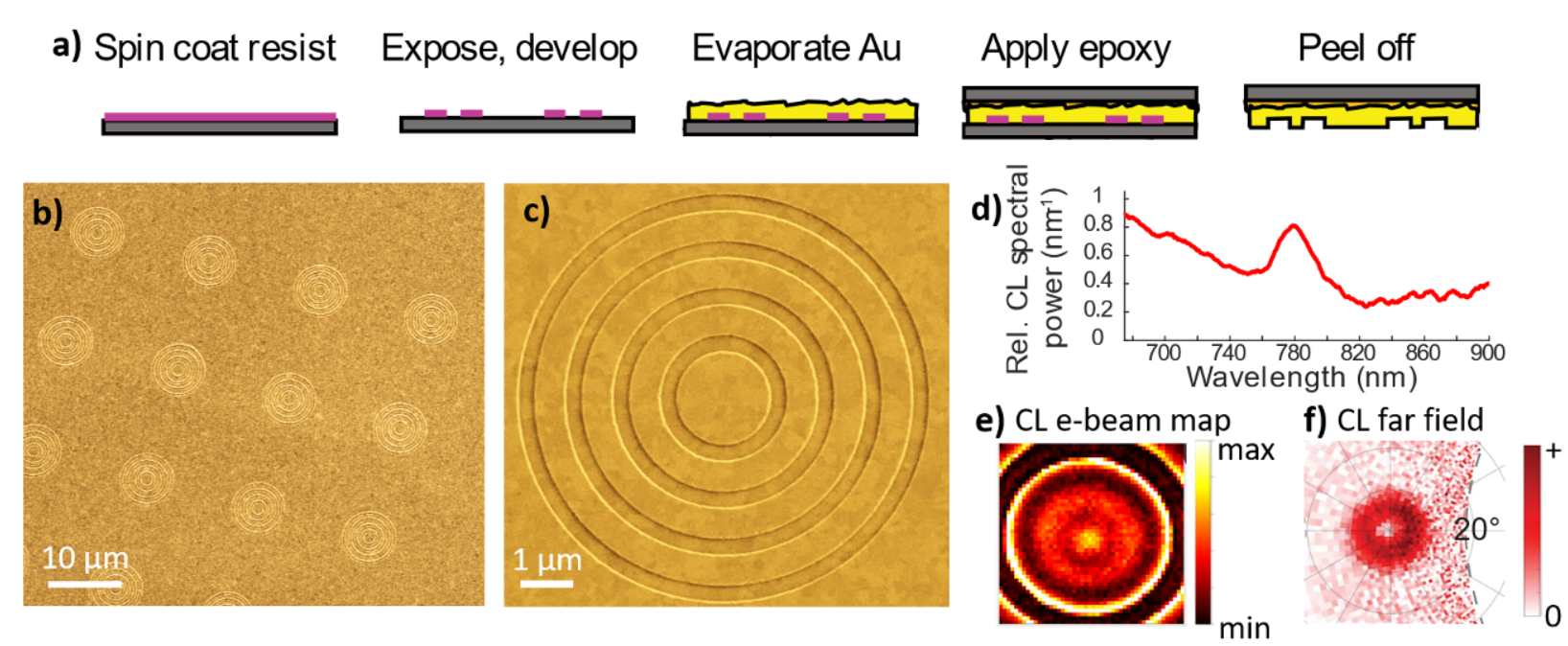

Figure 2. Fabrication of smooth plasmonic lens arrays and electro-optic characterization. (a) Schematic of fabrication steps, including electron-beam patterning of a mask in HSQ resist and template stripping of gold films. (b) Colorized in-lens SEM image of a portion of a gold plasmonic lens array. (c) Colorized in-lens SEM image of an individual plasmonic lens. (d) Cathodoluminescence (CL) spectrum measured while positioning a $20 \mathrm{kV}$ electron beam at the lens center. (e) Map of CL intensity collected within $10 \mathrm{~nm}$ of the peak wavelength in d as the electron beam is rastered across the central region of the lens. (f) Far field of CL emitted at $800 \mathrm{~nm}$ while electron beam is positioned at the lens center.

\section{PROTOTYPE FABRICATION AND ELECTRO-OPTICAL CHARACTERIZATION}

We have demonstrated production of mm-scale arrays of smooth plasmonic lens nanostructures using electronbeam lithography. Figure 2a shows a scanning electron microscope image of a few lenses from a chip containing hundreds of such lenses. We first produced a mask containing the bullseye nanopatterns in a negative electronbeam lithography resist consisting of $2 \%$ hydrogen silsesquioxane (HSQ) using the electron beam, then evaporated gold and peeled off to yield arrays of nanopatterned gold lenses. This template stripping process provides sub-nm 
roughness both on the top surface (0.4 nm RMS) and in the grooves (0.6 nm RMS) as confirmed using atomic force microscopy. The ability to make such large arrays of these structures opens up possible applications in multibeam tools.

We have also demonstrated high plasmonic mode quality and effective conversion from plasmons to light. This electro-optical characterization was achieved using cathodoluminescence (CL) spectromicroscopy in a scanning electron microscope, in which a focused electron beam locally excites radial surface plasmon polaritons and emitted light is collected using a parabolic mirror. A variety of spectral, spatial, and angle-resolved measurements were performed in a customized scanning electron microscope using the open-source ScopeFoundry platform. ${ }^{15,16}$ We found that optical emission was strongest near the $800 \mathrm{~nm}$ design wavelength, and mapping the dependence of that peak emission on electron beam position revealed a spatially smooth and symmetric plasmonic response. We also observed the emitted light was symmetric in the far field, and produced a vortex beam characteristic of radially polarized light. More details of the fabrication process and CL measurements can be found in ref. 11.

\section{DC PHOTOGUN FOR TESTING ULTRAFAST NANOEMITTERS}

To test and characterize next-generation photoemitters, we have built a DC photogun setup at Lawrence Berkeley National Laboratory (LBNL), shown in Figure 3. Up to $30 \mathrm{kV} \mathrm{DC}$ can be applied to the emitter as well as both rear and front optical illumination. The emitted electron beam first traverses a solenoid electromagnet, enabling focusing and solenoid-scan emittance measurement, followed by horizonal and vertical dipole steering electromagnets. Electron beams can be characterized by imaging using a phosphor screen or collecting them with a Faraday cup to measure photocurrent.

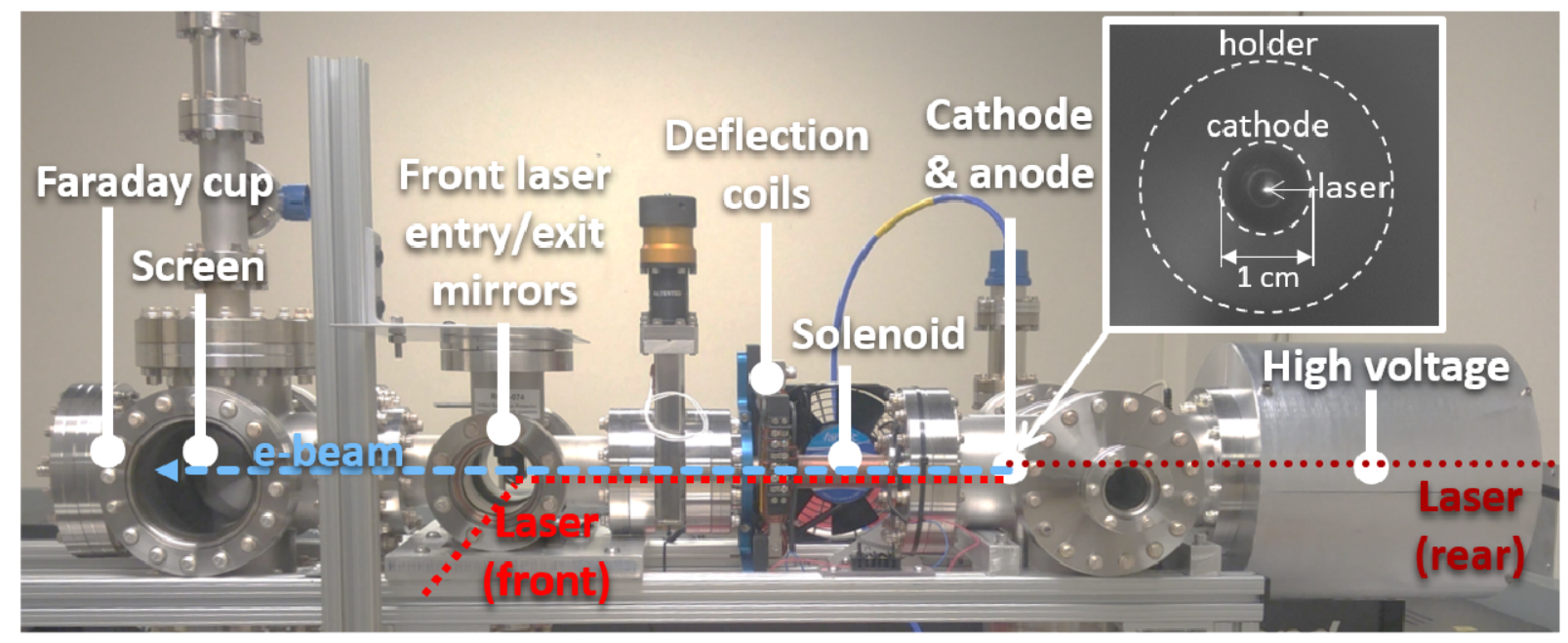

Figure 3. Photograph of DC photogun at LBNL with schematic labeling of components and beam paths. Inset shows an image viewed from the downstream end of a copper cathode mounted in the setup, including optical reflection from a laser focused on the cathode.

At present, the setup is equipped with a Femtolasers Ti:Sa oscillator well suited for ultrafast nanoemitters. The $80 \mathrm{MHz}$ repetition-rate allows to extract pA-level currents from single to few-electron emitters. This is important because positive charge left behind at the surface imposes a restoring field, limiting the number of electrons per pulse that can be extracted, especially in a low-voltage gun from a nanometric area. The formula for this space charge field is $E_{s c}=\frac{Q}{A \epsilon_{0}}$, where $\mathrm{Q}$ is the charge per pulse, A is the emission area, and $\epsilon_{0}$ is the vacuum permittivity. Using this, we can estimate the space charge field contributed by each additional electron within a pulse being extracted from a nanoemitter. Using the plasmonic lens described in section 2 as an example, we determine the emission area by approximating the emission as a flat-top distribution with radius equal to the half-width at half-maximum (about $71 \mathrm{~nm}$ ), giving an area of $1.6 \times 10^{4} \mathrm{~nm}^{2}$. Then, each electron extracted contributes $1.1 \mathrm{MV} / \mathrm{m}$. The DC gun applies at most $30 \mathrm{kV}$ across a $3 \mathrm{~mm}$ gap, providing a maximum 
field of $10 \mathrm{MV} / \mathrm{m}$. This suggests the emitted charge in this case will be limited to less than 10 electrons per pulse, or less than $125 \mathrm{pA}$ at $80 \mathrm{MHz}$ repetition rate. The limit on charge per pulse is independent of the pulse energy available, motivating the use of high repetition rate lasers and high extraction fields where high currents are needed from such nanoemitters.

For multiphoton photoemitters, high optical field intensity is crucial and so short pulse duration and small focal spots are an advantage. The Ti:Sa oscillator produces $500 \mathrm{~mW}$ of power with a bandwidth of about $87 \mathrm{~nm}$ FWHM and a pulse duration of less than 13.5 fs FWHM. We have so far achieved focal spots at the cathode as small as $71 \mu \mathrm{m}$ FWHM. While it is challenging to focus smaller with bulk optics given the geometry of the setup, particularly the distance between the laser entry viewport and photoemitter, plasmonic lens emitters help to overcome this limitation by concentrating the light incident over the entire lens area to a wavelength-scale central spot.
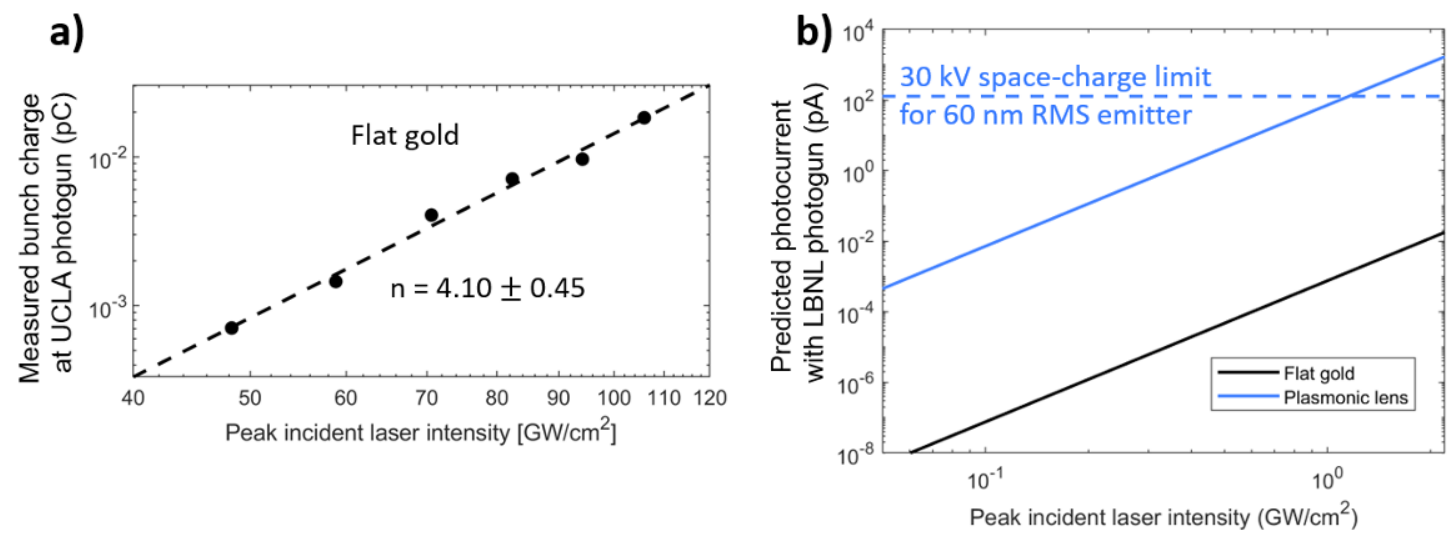

Figure 4. Determination of expected photocurrent from plasmonic lens nanoemitters in the LBNL DC photogun. (a) Measurement of multiphoton photoemission from a flat gold film on a similar setup at UCLA to verify 4-photon process and extract photoemission probability. (b) Calculations of expected photoemission at LBNL setup from a plasmonic lens compared to flat gold.

We can show that these laser parameters are sufficient to reach the space charge limit for the plasmonic lens nanoemitter described in Section 2. For an n-photon photoemission process, the charge per pulse Q is related to the optical pulse parameters by $Q=C A t I^{n}=C \frac{E^{n}}{\tau^{n-1} A^{n-1}}$ where I is the peak optical intensity, E is the optical pulse energy, $\tau$ is the optical intensity duration $\left(\sqrt{2 \pi} \sigma_{t}\right.$ for a Gaussian pulse, where $\sigma_{t}$ is RMS pulse duration), A is the optical intensity spot area ( $A=2 \pi \sigma^{2}$ for a Gaussian beam, where $\sigma$ is the RMS spot size), and C is a wavelength- and material-dependent proportionality constant. ${ }^{17}$ To determine $\mathrm{C}$ for $800 \mathrm{~nm}$ wavelength light on flat gold, we performed photoemission measurements at a similar DC photogun at University of California, Los Angeles (UCLA). These measurements used an amplified Ti:Sa laser with about $50 \mu \mathrm{m}$ RMS spot size, $100 \mathrm{fs}$ FWHM pulse duration, and incident pulse energy varied from $0.8 \mu \mathrm{J}$ to $1.8 \mu \mathrm{J}$. we were able to verify a 4-photon process by performing a power law fit to $\mathrm{Q}$ as a function of I, shown in Figure 4a. Then, by fixing $\mathrm{n}$ to be 4 and fitting the curve again (not shown in the plot), we determine $C=10^{-5.02 \pm 0.02} \frac{\mathrm{A}}{\mathrm{cm}^{2}} /\left(\frac{\mathrm{GW}}{\mathrm{cm}^{2}}\right)^{4}$.

We then calculated the expected photocurrent for flat gold and the 4-ring gold plasmonic lens for the LBNL setup. Photocurrent, $\mathrm{i}$, is given by $i=f_{r e p} Q$, where $f_{r e p}$ is the repetition rate ( $80 \mathrm{MHz}$ for our laser) and $\mathrm{Q}$ is the charge per pulse. We consider a range of pulse energies from $0.02 \mathrm{~nJ}$ to $2 \mathrm{~nJ}$ (about a third of the full laser power). For the flat gold, we consider the laser parameters discussed above to compute Q: $71 \mu \mathrm{m}$ FWHM spot size and 15 fs pulse duration, assuming optimal focusing and pulse compression at the cathode. The plasmonic lens, however, modifies the optical intensity profile at the surface, focusing the RMS spot size to $\sigma=161 \mathrm{~nm}$ and increasing temporal duration by about $4 \mathrm{fs}$ (see ref. 11). In doing so, the peak optical field is enhanced by about 15 times (for 15 fs incident laser pulse, see ref. 11). The optical pulse energy concentrated into the lens center is calculated using $E_{\text {nano }}=F^{2} A_{\text {nano }} t_{\text {nano }} I_{\text {inc }}$ where $\mathrm{F}$ is the field enhancement factor, nano subscripts refer to the nanofocused optical intensity, and $I_{i n c}$ is the peak incident laser intensity. We find that only $0.84 \%$ of the incident 
laser pulse energy is coupled into a single plasmonic lens in this case, mainly because the incident beam is more than 10 times larger in diameter than the 4-ring bullseye lens. The predicted photocurrent as a function of the laser power is shown in Figure 4b. We predict nearly 100,000 times greater photocurrent from the plasmonic structure compared to the flat gold, allowing to characterize the emission from the structure with negligible photoemitted background current from surrounding areas. Furthermore, these calculations suggest that with our laser parameters, we should be able to achieve the maximum allowed photocurrent from the plasmonic lens nanoemitter at $30 \mathrm{kV}$ and measure the onset of virtual cathode formation.

We note that higher total photocurrent could be obtained by measuring an array of lenses simultaneously. In such a case, a high degree of reproducibility as afforded by the electron-beam lithography method discussed in section 3 is crucial for accurate characterization. Meanwhile for a single lens, the photocurrent can be enhanced by increasing the number of rings, thereby coupling more incident laser power into the structure. However, this comes at the expense of longer response time (more time delay between SPPs generated at inner and outer rings, see ref. 11) and the maximum photocurrent is still set by the space-charge limit.

\section{CONCLUSIONS}

Plasmon-enhanced photoemitters, like the plasmonic lens design discussed here, provide a design paradigm for tuning spatial and temporal characteristics of electron beams. Our plasmonic lens design provides an example of fixed, nanometric spot size with fast temporal response, allowing imprinting of temporal characteristics of the laser onto the electron beam. Future designs could further modify the spatial or temporal characteristics of the optical field at the surface to create spatially complex beams, such as multibeam arrays, with varying temporal relationships between the beams. Other designs could utilize plasmonic enhancement to access the optical field emission regime ${ }^{18}$ and enable photoemitters that take advantage of effects like phase control in fewcycle pulses. ${ }^{19}$ A DC photogun like ours could be used to characterize a broad range of ultrafast nanoemitters, ranging from spatial and angular characteristics of the beams to quantum yield. We anticipate this work will set up a succession of ultrafast photoemitter developments and enable new scientific and industrial capabilities for electron beams.

\section{ACKNOWLEDGMENTS}

D.F. and the work at the Molecular Foundry were supported by the Office of Science, Office of Basic Energy Sciences, of the U.S. Department of Energy under Contract No. DE-AC02-05CH11231. Funding for D.B.D. and the collaboration with UCLA was provided by STROBE: A National Science Foundation Science and Technology Center under Grant No. DMR 1548924. We thank Russell P. Wells for engineering contributions to design and construction of the DC photogun at LBNL; Scott Dhuey for assistance with electron-beam lithography of plasmonic lenses; and D. Frank Ogletree, Edward S. Barnard, and Shaul Aloni for assistance with the cathodoluminescence spectromicroscopy measurements.

\section{REFERENCES}

[1] Feist, A., Bach, N., da Silva, N. R., Danz, T., Möller, M., Priebe, K. E., Domröse, T., Gatzmann, J. G., Rost, S., Schauss, J., et al., "Ultrafast transmission electron microscopy using a laser-driven field emitter: Femtosecond resolution with a high coherence electron beam," Ultramicroscopy 176, 63-73 (2017).

[2] Ji, F., Durham, D. B., Minor, A. M., Musumeci, P., Navarro, J. G., and Filippetto, D., "Ultrafast relativistic electron nanoprobes," Communications Physics 2(1), 54 (2019).

[3] Sannibale, F., Filippetto, D., Papadopoulos, C., Staples, J., Wells, R., Bailey, B., Baptiste, K., Corlett, J., Cork, C., De Santis, S., et al., "Advanced photoinjector experiment photogun commissioning results," Physical Review Special Topics-Accelerators and Beams 15(10), 103501 (2012).

[4] Pan, L., "Complementary patterning using plasmon-excited electron beamlets," in [2016 Progress in Electromagnetic Research Symposium (PIERS)], 2663-2663, IEEE (2016).

[5] Vogelsang, J., Robin, J., Nagy, B. J., Dombi, P., Rosenkranz, D., Schiek, M., Groß, P., and Lienau, C., "Ultrafast electron emission from a sharp metal nanotaper driven by adiabatic nanofocusing of surface plasmons," Nano Letters 15(7), 4685-4691 (2015). 
[6] Polyakov, A., Cabrini, S., Dhuey, S., Harteneck, B., Schuck, P., and Padmore, H., "Plasmonic light trapping in nanostructured metal surfaces," Applied Physics Letters 98(20), 203104 (2011).

[7] Polyakov, A., Senft, C., Thompson, K., Feng, J., Cabrini, S., Schuck, P., Padmore, H., Peppernick, S. J., and Hess, W. P., "Plasmon-enhanced photocathode for high brightness and high repetition rate x-ray sources," Physical review letters 110(7), 076802 (2013).

[8] Li, R., To, H., Andonian, G., Feng, J., Polyakov, A., Scoby, C., Thompson, K., Wan, W., Padmore, H., and Musumeci, P., "Surface-plasmon resonance-enhanced multiphoton emission of high-brightness electron beams from a nanostructured copper cathode," Physical review letters 110(7), 074801 (2013).

[9] Raether, H., "Surface plasmons on gratings," in [Surface plasmons on smooth and rough surfaces and on gratings], 91-116, Springer (1988).

[10] Steele, J. M., Liu, Z., Wang, Y., and Zhang, X., "Resonant and non-resonant generation and focusing of surface plasmons with circular gratings," Optics Express 14(12), 5664-5670 (2006).

[11] Durham, D. B., Riminucci, F., Ciabattini, F., Mostacci, A., Minor, A. M., Cabrini, S., and Filippetto, D., "Plasmonic lenses for tunable ultrafast electron emitters at the nanoscale," Physical Review Applied 12(5), 054057 (2019).

[12] Chen, W., Abeysinghe, D. C., Nelson, R. L., and Zhan, Q., "Plasmonic lens made of multiple concentric metallic rings under radially polarized illumination," Nano letters $\mathbf{9}(12), 4320-4325$ (2009).

[13] Lerman, G. M., Yanai, A., and Levy, U., "Demonstration of nanofocusing by the use of plasmonic lens illuminated with radially polarized light," Nano letters 9(5), 2139-2143 (2009).

[14] Lumerical, "FDTD solutions." http://www.lumerical.com.

[15] Barnard, E. S., "ScopeFoundry: A python platform for controlling custom laboratory experiments and visualizing scientific data." http://www.scopefoundry.org/.

[16] Durham, D. B., Ogletree, D. F., and Barnard, E. S., "Scanning Auger spectromicroscopy using the ScopeFoundry software platform," Surface and Interface Analysis 50(11), 1174-1179 (2018).

[17] Musumeci, P., Cultrera, L., Ferrario, M., Filippetto, D., Gatti, G., Gutierrez, M., Moody, J., Moore, N., Rosenzweig, J., Scoby, C., et al., "Multiphoton photoemission from a copper cathode illuminated by ultrashort laser pulses in an rf photoinjector," Physical review letters 104(8), 084801 (2010).

[18] Krüger, M., Schenk, M., Förster, M., and Hommelhoff, P., "Attosecond physics in photoemission from a metal nanotip," Journal of Physics B: Atomic, Molecular and Optical Physics 45(7), 074006 (2012).

[19] Piglosiewicz, B., Schmidt, S., Park, D. J., Vogelsang, J., Groß, P., Manzoni, C., Farinello, P., Cerullo, G., and Lienau, C., "Carrier-envelope phase effects on the strong-field photoemission of electrons from metallic nanostructures," Nature Photonics 8(1), 37 (2014). 